\title{
Eternal discrete time crystal beating the Heisenberg limit
}

\author{
Changyuan Lyu $\odot,{ }^{1}$ Sayan Choudhury, ${ }^{1}$ Chenwei Lv $\odot,{ }^{1}$ Yangqian Yan, ${ }^{1,2, *}$ and Qi Zhou ${ }^{1,2,3, \dagger}$ \\ ${ }^{1}$ Department of Physics and Astronomy, Purdue University, 525 Northwestern Avenue, West Lafayette, Indiana 47907, USA \\ ${ }^{2}$ Center for Science of Information, Purdue University, West Lafayette, Indiana 47907, USA \\ ${ }^{3}$ Purdue Quantum Science and Engineering Institute, Purdue University, 1205 West State Street, West Lafayette, Indiana 47907, USA
}

(Received 23 August 2019; accepted 28 June 2020; published 14 July 2020)

\begin{abstract}
A discrete time crystal (DTC) repeats itself with a rigid rhythm, mimicking a ticking clock set by the interplay between its internal structures and an external force. Discrete time crystals promise profound applications in precision timekeeping and other quantum techniques. However, it has been facing a grand challenge of thermalization. The periodic driving supplying the power may ultimately bring DTCs to thermal equilibrium and destroy their coherence. Here we show that an all-to-all interaction delivers a DTC that evades thermalization and maintains quantum coherence and quantum synchronization regardless of spatial inhomogeneities in the driving field and the environment. Moreover, the sensitivity of this DTC scales with the total particle number to the power of $3 / 2$, realizing a quantum device of measuring the driving frequency or the interaction strength beyond the Heisenberg limit. Our work paves the way for designing nonequilibrium phases with long coherence time to advance quantum metrology.
\end{abstract}

DOI: 10.1103/PhysRevResearch.2.033070

\section{INTRODUCTION}

A periodic driving continuously pumps energies into a discrete time crystal (DTC) [1-15] and eventually heats it up to infinite temperature [16-18]. Many schemes have been proposed to slow down thermalization [5-7,10], including many-body localization (MBL), Floquet prethermalization, and cryptoequilibrium. Compared with other schemes retaining the coherence of DTCs within certain timescales, MBL is of particular interest. Disorder breaks an interacting system into localized $l$-bits [19], suppressing thermalization up to an arbitrarily long timescale. However, most studies so far have considered homogeneous drivings. In practice, the driving field may vary across a DTC and local perturbations may further amplify spatial inhomogeneities, both preventing constituents of the DTC from synchronization and impeding applying DTCs in quantum technologies. Whereas MBL could stabilize a DTC against weak inhomogeneous perturbations to $\pi$ rotations [20], it is no longer powerful in the presence of strong inhomogeneities, as exponentially decayed couplings between $l$-bits in MBL have readily weakened the synchronization between remote parts. Fundamental questions naturally arise: how to access a DTC that could maintain quantum coherence and synchronization in the presence of arbitrarily strong inhomogeneities of driving fields and local

\footnotetext{
*yan281@purdue.edu

†zhou753@purdue.edu
}

Published by the American Physical Society under the terms of the Creative Commons Attribution 4.0 International license. Further distribution of this work must maintain attribution to the author $(s)$ and the published article's title, journal citation, and DOI. perturbations and how to implement such a DTC to promote the precision of quantum metrology.

In this paper we present a type of DTC that has a number of unique features distinct from previously studied ones. Discrete time crystals in the literature survive a small deviation of the driving fields from uniform $\pi$ pulses. In contrast, our DTC is stable against arbitrarily strong perturbations in both homogeneous and inhomogeneous pulses. It could also start from any initial state, not necessarily a superposition of only two eigenstates of the Floquet operator. Meanwhile, the hypersensitivity of our DTC to interaction strength makes it a promising quantum device to measure interactions beyond the Heisenberg limit, unlike other DTCs not sensitive to interactions within a finite range.

\section{MODEL}

We consider $N$ spins $1 / 2$ described by a Hamiltonian $H=$ $H_{\text {int }}+\sum_{n} H_{\text {pul }} \delta(t-n T)$, where

$$
H_{\mathrm{int}}=2 J \sum_{i<j} S_{i}^{z} S_{j}^{z}, \quad H_{\mathrm{pul}}=\sum_{i}^{N} \theta_{i} S_{i}^{y} .
$$

As shown in Fig. 1(a), $J$ is the strength of an all-to-all interaction considered in the Lipkin-Meshkov-Glick model [21]. In addition, $\vec{S}_{i}=\frac{1}{2} \vec{\sigma}_{i}$, where $\vec{\sigma}_{i}$ are Pauli matrices $(\hbar=1), H_{\text {pul }}$ represents periodic pulses applied on spins, and $\theta_{i}$ determines the angle rotated by the $i$ th spin about the $y$ axis. The dependence of $\theta_{i}$ on $i$ characterizes the spatial inhomogeneity of pulses. In the time evolution, $H_{\text {int }}$ and $H_{\text {pul }}$ control the dynamical phases and the geometric phases, respectively, both of which depend on the numbers of spin excitations. Since $H_{\text {int }}$ and $H_{\text {pul }}$ do not commute, their interplay will lead to a perfect revival of the initial state. Equation (1) can be realized using spins $1 / 2$ coupled to a cavity or a waveguide $[22,23]$ or 


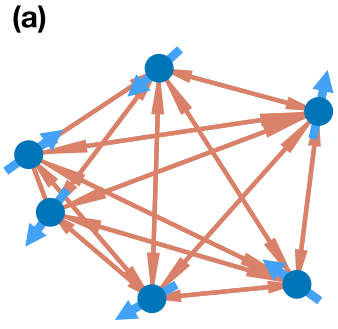

(b) $\quad\left|\Psi\left(0^{-}\right)\right\rangle$

\section{(c) $2 n T^{-}$}

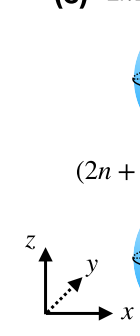

$\left|\Psi\left(T^{-}\right)\right\rangle$

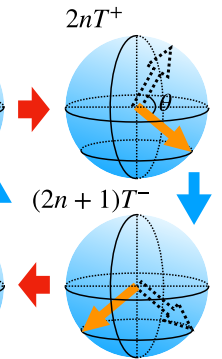

$\left|\Psi\left(2 T^{-}\right)\right\rangle$

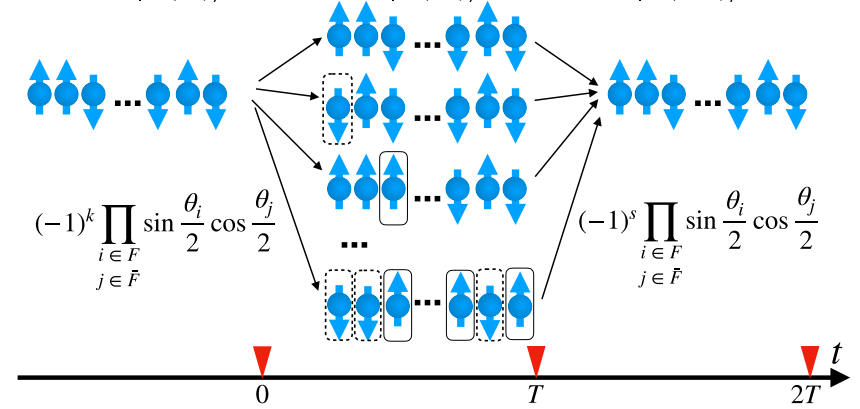

FIG. 1. (a) All-to-all interactions (brown arrows) between spin $1 / 2$ (blue spheres attached to arrows). (b) Perfect revival of an arbitrary initial state due to constructive interference among all pathways. Dashed and solid boxes highlight the $k$ spins up and $s$ spins down flipped by the first pulse, leading to geometric phases $(-1)^{k}$ and $(-1)^{s}$, respectively. Triangles on the time axis represent $H_{\text {pul }}$. (c) Rotations of a spin $L$ (yellow arrow) on the Bloch sphere. When $J T=\pi$, the nonlinear term $J L_{z}^{2}$ leads to an effective $\pi$ rotation about the $z$ axis between $2 n T^{+}$and $(2 n+1) T^{-}$such that any initial state returns to itself after $2 T$ for any $\theta$.

particles with long-range interactions whose ranges are much larger than the system size. The equivalence between spins $1 / 2$ and bosons also provides a natural realization [24].

We prove that, when $J T=\pi$, any initial state returns to itself at $t=2 n T^{-}$for any $N \in 2 \mathbb{Z}$ and $\theta_{i}$ as an arbitrary function of $i$. Here $t^{-}\left(t^{+}\right)$denotes the time right before (after) applying a pulse. This perfect revival delivers an eternal DTC evading thermalization and equipped with a strong synchronization even in a noisy environment. Previous works on normalized all-to-all interactions have considered the small- $J$ limit of Eq. (1) [24], not the optimal choice of $J T$ discussed here.

Consider an initial state with $m$ spins up and $N-m$ spins down $\left|\Psi\left(0^{-}\right)\right\rangle=\prod_{i}|\eta\rangle_{i}$, where $\eta=\uparrow, \downarrow$. After the first pulse,

$$
\begin{aligned}
& |\uparrow\rangle_{i} \rightarrow+\cos \left(\frac{\theta_{i}}{2}\right)|\uparrow\rangle_{i}+\sin \left(\frac{\theta_{i}}{2}\right)|\downarrow\rangle_{i}, \\
& |\downarrow\rangle_{i} \rightarrow-\sin \left(\frac{\theta_{i}}{2}\right)|\uparrow\rangle_{i}+\cos \left(\frac{\theta_{i}}{2}\right)|\downarrow\rangle_{i} .
\end{aligned}
$$

Here $\left|\Psi\left(0^{+}\right)\right\rangle$becomes a superposition of $2^{N}$ states. Each state is obtained from flipping $s$ spins up and $k$ spins down of $\left|\Psi\left(0^{-}\right)\right\rangle$, as shown in Fig. 1(b). Each state acquires a dynamical phase $e^{-i \varphi_{1}}$ imposed by $H_{\text {int }}$ from $t=0^{+}$to $t=T^{-}$. The second pulse flips spins again, followed by $H_{\text {int }}$ imposing another dynamical phase $e^{-i \varphi_{2}}$ from $t=T^{+}$to $t=2 T^{-}$. We define $A=\left\langle\Psi\left(0^{-}\right)\right| \Psi\left(2 T^{-}\right)$.
To return to $\left|\Psi\left(0^{-}\right)\right\rangle$, the $s(k)$ spins up (down) flipped by the first pulse need to flip back to spins up (down) during the second pulse; $2^{N}$ such pathways allow the system to return to $\left|\Psi\left(0^{-}\right)\right\rangle$. The contribution to $A$ from each pathway is $(-1)^{k+s} \prod_{j \in \bar{F}} \cos ^{2}\left(\frac{\theta_{j}}{2}\right) \prod_{i \in F} \sin ^{2}\left(\frac{\theta_{i}}{2}\right)$, where $(-1)^{k+s}$ comes from flipping $k+s$ spins $1 / 2$ twice, equivalent to the geometric phase from rotating these spins about the $y$ axis for $2 \pi$. Here $F(\bar{F})$ denotes the collection of flipped (unflipped) spins. As each of these $2^{N}$ states is an eigenstate of $H_{\text {int }}, \varphi_{1}=$ $(m-s+k)(m-s+k-N) \pi$ and $\varphi_{2}=m(m-N) \pi$ when $J T=\pi$. The $m$-independent terms have been dropped. Since $N \in 2 \mathbb{Z}$ and $e^{i Z^{2} \pi}=(-1)^{Z} \forall Z \in \mathbb{Z}$, we have $e^{-i\left(\varphi_{1}+\varphi_{2}\right)}=$ $e^{i \pi\left\{2\left[m^{2}+m(k-s-N)-k s\right]+N(s-k)+k^{2}+s^{2}\right\}}=(-1)^{k+s}$, which cancels the previously obtained geometric phase, and thus $A=$ $\sum_{F} \prod_{j \in \bar{F}} \cos ^{2}\left(\frac{\theta_{j}}{2}\right) \prod_{i \in F} \sin ^{2}\left(\frac{\theta_{i}}{2}\right)$. Since $\sum_{F}$ denotes the sum over all $2^{N}$ choices of flipping the $N$ spins in $\left|\Psi\left(0^{-}\right)\right\rangle$,

$$
A=\prod_{i}\left(\sin ^{2} \frac{\theta_{i}}{2}+\cos ^{2} \frac{\theta_{i}}{2}\right)=1 .
$$

This discussion applies to any initial product state and any $t \in\left[2 n T^{-}, 2(n+1) T^{-}\right]$. Thus, any initial state returns to itself at $t=2 n T^{-}$. Unlike traditional spin-echo schemes using tailored pulses [25], we implement interactions, one source of the decoherence, to overcome the other, the inhomogeneities, so as to access a perfect dynamical localization, an analogy to the Anderson localization in the Hilbert space [26]. This interaction-induced spin echo applies to a broad class of systems to extend the coherence time.

For spatially uniform pulses, a simpler proof exists. Here $H$ is rewritten as

$$
H_{\text {hom }}=J L_{z}^{2}+\theta L_{y} \sum_{n} \delta(t-n T),
$$

where $\vec{L}=\sum_{i} \vec{S}_{i}$. Equation (5) is equivalent to the kicked top model for spin $L$ [27], where $L=\frac{N}{2}$. The propagator from $t=2 n T^{-}$to $t=2(n+1) T^{-}$is $U_{J T}(2 T)=e^{-i J T L_{z}^{2}} e^{-i \theta L_{y}} e^{-i J T L_{z}^{2}} e^{-i \theta L_{y}} . \quad$ As $e^{-i \pi L_{z}^{2}}=e^{-i \pi L_{z}}$ applies to any integer $L$ (or even $N$ ), $U_{\pi}(2 T)=$ $e^{-2 i \pi L_{z}} e^{e^{i \pi L_{z}}\left(-i \theta L_{y}\right) e^{-i \pi L_{z}}} e^{-i \theta L_{y}}=1$. As shown in Fig. 1(c), any state on the Bloch sphere returns to the original place after $2 T$. If $N \in 2 \mathbb{Z}+1, e^{-i \pi L_{z}^{2}}$ and $e^{-i \pi L_{z}}$ are no longer identical, and such a DTC with a period of $2 T$ does not exist. In contrast, if we consider spin 1 in Eq. (1), such an even-odd effect is absent, since $L \in \mathbb{Z} \forall N$.

Here $U_{\pi}(2 T)=1$ means that the quasienergy spectrum of

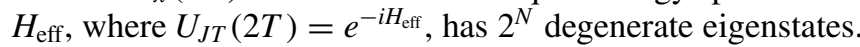
Whereas this looks similar to the noninteracting case when $\theta_{i}=\pi$, a conceptual difference is that the degeneracy here is stable against any perturbations in $\theta_{i}$, unlike noninteracting systems, where any infinitesimal deviation from a homogeneous $\pi$ pulse lifts the degeneracy, breaks the integrability, and suppresses DTCs. Similar to other models, the period doubling comes from the spontaneous time-translation symmetry breaking $[5,6,20,24]$. When $J T=\pi$, the Floquet eigenstates are $| \pm\rangle=\frac{1}{\sqrt{2}}|\psi(0)\rangle \pm \frac{1}{\sqrt{2}} U_{\pi}(T)|\psi(0)\rangle$, where $|\psi(0)\rangle$ is an arbitrary initial state. Equivalently, every state $|+\rangle$ with quasienergy 0 has a partner $|-\rangle$ with quasienergy $\pi / T$. Choosing the initial state as $|\psi(0)\rangle=\frac{1}{\sqrt{2}}(|+\rangle+|-\rangle)$, the 

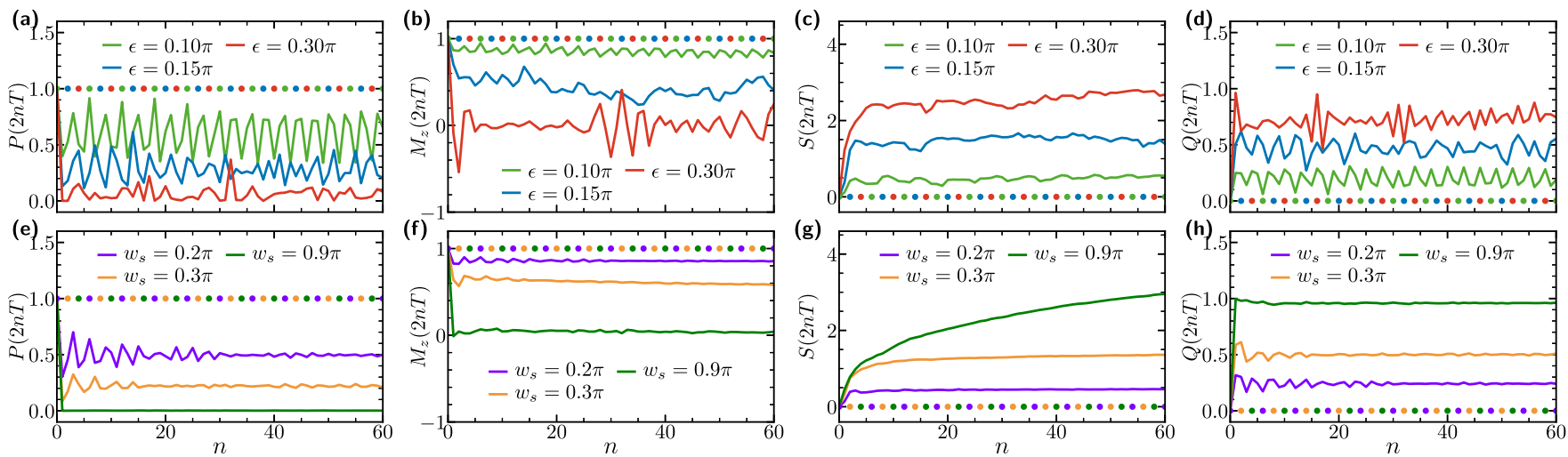

FIG. 2. Comparison between the all-to-all interaction and a power-law potential with $\alpha=3$. Here $N=14$. (a)-(d) Uniform pulses, with $w_{s}=0$. The DTC with all-to-all interactions (dots) is unaffected by the deviation $\epsilon$. With the power-law potential (curves), increasing $\epsilon$ leads to the suppression of $P(2 n T)$ and $M_{z}(2 n T)$ and the growth of $S(2 n T)$ and $Q(2 n T)$. (e)-(h) Keeping $\bar{\theta}=\pi$ and increasing the spatial inhomogeneities $w_{s}$, the DTC with the power-law potential is suppressed. The DTC with all-to-all interactions remains stable. See Appendix B for more results of $\bar{\theta} \neq \pi$.

Rabi oscillation between the two Floquet eigenstates leads to a period of $2 T$. Previous works have mainly focused on uniform $\pi$ pulses, where the Floquet eigenstate $\frac{1}{\sqrt{2}}|\uparrow \uparrow \cdots\rangle \pm \frac{1}{\sqrt{2}} \mid \downarrow \downarrow$ $\cdots\rangle$ is a special case of our results. In particular, results here apply to any uniform and nonuniform pulses and thus are far more general.

\section{STABILITY AGAINST SPATIAL INHOMOGENEITIES}

We compare our model to the power-law interaction $H^{\prime}=$ $H_{\text {int }}^{\prime}+\sum_{n} H_{\text {pul }} \delta(t-n T)$, where

$$
H_{\mathrm{int}}^{\prime}=2 J \sum_{i<j} \frac{S_{i}^{z} S_{j}^{z}}{|i-j|^{\alpha}} .
$$

Starting from $\left|\Psi\left(0^{-}\right)\right\rangle=\prod_{i}|\uparrow\rangle_{i}$, we compute

$$
\begin{aligned}
P\left(2 n T^{-}\right) & =\left|\left\langle\Psi\left(0^{-}\right)|| \Psi\left(2 n T^{-}\right)\right\rangle\right|^{2}, \\
M_{z}\left(2 n T^{-}\right) & =2\left\langle\Psi\left(2 n T^{-}\right)\left|L_{z}\right| \Psi\left(2 n T^{-}\right)\right\rangle / N, \\
E\left(2 n T^{-}\right) & =\left\langle\Psi\left(2 n T^{-}\right)\left|H_{\mathrm{int}}\right| \Psi\left(2 n T^{-}\right)\right\rangle, \\
S\left(2 n T^{-}\right) & =-\operatorname{Tr}\left(\rho_{B} \ln \rho_{B}\right) .
\end{aligned}
$$

Here $P\left(2 n T^{-}\right)$characterizes the quantum memory of the initial state, $M_{z}\left(2 n T^{-}\right)$denotes the $z$ component of the total spin, $E\left(2 n T^{-}\right)$[or $E^{\prime}\left(2 n T^{-}\right)=\left\langle\Psi\left(2 n T^{-}\right)\left|H_{\text {int }}^{\prime}\right| \Psi\left(2 n T^{-}\right)\right\rangle$] captures the absorption of energy, and $S\left(2 n T^{-}\right)$is the halfchain entanglement entropy.

When $\theta_{i}=\bar{\theta}$, a finite $J$ in Eq. (6) restores the quantum coherence, if $\epsilon=\bar{\theta}-\pi$ is small [7-9]. With increasing $\epsilon$, $P\left(2 n T^{-}\right)$and $M_{z}\left(2 n T^{-}\right)$get suppressed [Figs. 2(a)-2(d)]. Meanwhile, $Q$ and $S$ grow quickly, where $Q=\frac{E\left(2 n T^{-}\right)-E(0)}{E_{\infty}-E(0)}$ characterizes the absorption of the energy. In addition, $E_{\infty}=$ $2^{-N} \sum_{j}\left\langle j\left|H_{\text {int }}\right| j\right\rangle$ is the energy at infinite temperature and $\sum_{j}$ sums over all eigenstates of $H_{\text {int }}$. These results signify the thermalization at large $\epsilon$. Taking into account spatial inhomogeneities, as shown in Figs. 2(e)-2(h), we choose a random $\theta_{i}$ from $\left[\bar{\theta}-w_{s}, \bar{\theta}+w_{s}\right]$ with a constant probability. When $w_{s}$ is finite, the thermalization becomes even faster and $Q$ approaches 1 , indicating thermalization to infinite temperature. For power-law interactions, dynamical phases controlled by interactions cannot cancel geometric phases induced by pulses. It is impossible to obtain a constructive interference between all pathways. Whereas on-site disorders are introduced to create MBL to slow down the thermalization [7-9], an intrinsic drawback is that MBL weakens the synchronization when the pulses are nonuniform (Appendix A). In contrast, $P\left(2 n T^{-}\right)$and $M_{z}\left(2 n T^{-}\right)$of the all-to-all interaction are unaffected by $w_{s}$, and $Q\left(2 n T^{-}\right)$and $S\left(2 n T^{-}\right)$remain zero, directly reflecting the robustness of this eternal DTC.

\section{APPLICATIONS}

The perfect revival at $t=2 n T^{-}$comes from the same phase of all $2^{N}$ pathways of returning to $|\Psi(0)\rangle$ when $J T=\pi$. Once $J T \neq \pi$, the larger $N$ is, the more rapidly the phase varies with changing pathways. In the large- $N$ limit, this DTC becomes supersensitive to $J T$ and serves as a high-precision device to measure either $J$ or $T$.

It is time consuming to solve more than 14 lattice sites using exact diagonalization when inhomogeneities exist. We focus on homogeneous systems. It is expected that the lower bound of the results of an inhomogeneous distribution $\theta_{i} \in$ $\left[\bar{\theta}-w_{s}, \bar{\theta}+w_{s}\right]$ could be estimated using homogeneous $\theta_{i}=$ $\bar{\theta} \pm w_{s}$. As an example, we consider $\theta_{i}$ fixed at $\pi / 4$. As shown in Fig. 3(a), $P\left(2 n T^{-}\right)$quickly vanishes if $|\delta| \gg \pi / N^{3 / 2}$, where $\delta=J T-\pi$. Whereas using highly entangled states in linear interferometry could beat the standard quantum limit $1 / \sqrt{N}$ and access the Heisenberg limit $1 / N$, nonlinear interferometry using $k$-body interactions could beat the Heisenberg limit and reach the ultimate limit $N^{-k}$ [28-33]. Our DTC represents a different category of nonlinear metrology using periodic drivings to beat the Heisenberg limit.

The $P(2 T)$ captures short-time dynamics. Figure $3(\mathrm{c})$ shows that the dependence of $P(2 T)$ on $J T$ has a narrow peak centered at $\pi$, whose width $\sim 1 / N^{3 / 2}$. Such scaling can be obtained analytically (Appendix C 1 ) and is verified numerically [inset of Fig. 3(c)]. The power spectrum $\tilde{P}(f)=$ $\frac{1}{M} \sum_{n=0}^{M-1} e^{i 2 \pi n T f} P(n T)$ contains information about both shortand long-time dynamics. Here $\tilde{P}(1 / 2 T)$ characterizes the 

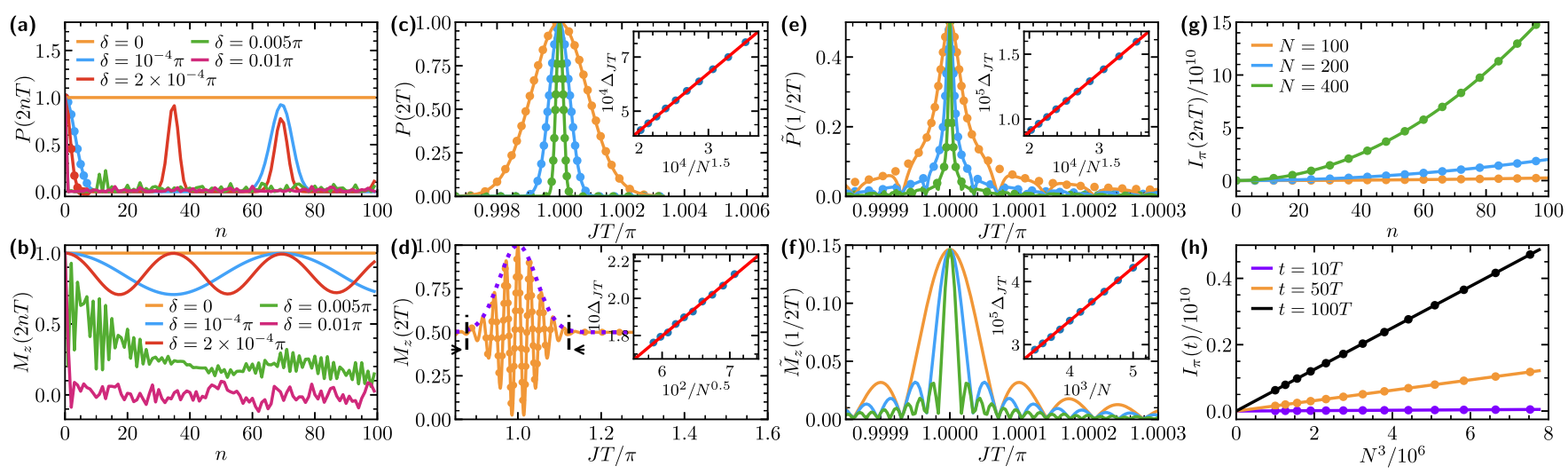

FIG. 3. Sensitivity to $J T$. Curves (dots) are numerical (analytical) results. Orange, blue, and green colors in (c)-(f) represent $N=100$, 200, and 400, respectively. (a) $P(2 n T)$ and (b) $M_{z}(2 n T)$ as functions of $n$ at various $J T$. When $|J T-\pi| \gg \pi / N^{3 / 2}$, both quantities quickly decrease down to zero. Here $N=200$ has been used in the calculation. (c) $P(2 T)$ and (d) $M_{z}(2 T)$ as a function of $J T$. For a fixed $N, P(2 T)$ and $M_{z}(2 T)$ have narrow peaks centered at $J T=\pi$. In contrast, $M_{z}(2 T)$ has an additional fast oscillation; the dashed curve highlights the analytical result of its profile, whose width is denoted by black arrows. (e) $\tilde{P}(1 / 2 T)$ and (f) $\tilde{M}_{z}(1 / 2 T)$ are also featured by narrow peaks around $J T=\pi$. Here $M=200$ is used in numerics. Insets in (c)-(f) show scalings of widths of peaks with $N$. (g) Quantum Fisher information $I_{\pi}(2 n T)$ as a function of $n$. (h) $I_{\pi}(2 n T)$ is proportional to $N^{3}$. The value $\theta_{i}=\pi / 4$ is used in all panels.

response at half of the frequency of the periodic driving. Its dependence on $J T$ also has a peak around $\pi$. We define the full width at half maximum as $\Delta_{J T}$ and find both numerically and analytically that $\Delta_{J T}$ is proportional to $1 / N^{3 / 2}$ (Appendix C 1).

We also consider the quantum Fisher information [34]

$$
\begin{aligned}
I_{J T}(2 n T) & =\lim _{\epsilon \rightarrow 0} 4 \frac{1-F_{\epsilon}}{\epsilon^{2}}, \\
F_{\epsilon} & =\left|\left\langle\Psi\left(0^{-}\right)\left|U_{J T}(2 n T) U_{J T+\epsilon}(-2 n T)\right| \Psi\left(0^{-}\right)\right\rangle\right|^{2},
\end{aligned}
$$

where $F_{\epsilon}$ is the Loschmidt echo. The uncertainty of $J T$ is bounded by $\sqrt{I_{J T}(2 n T)}$, i.e., $\Delta_{J T} \geqslant 1 / \sqrt{I_{J T}(2 n T)}$. We have found analytically that (Appendix D)

$$
I_{\pi}(2 n T)=\frac{n^{2}}{4}\left[\sin ^{2}(2 \bar{\theta}) N^{3}+2 \sin ^{4}(\bar{\theta}) N^{2}\right] .
$$

When $\quad \bar{\theta} \neq 0, \pm \pi / 2, \pi, \quad I_{\pi}(2 n T) \sim n^{2} N^{3}, \quad$ provided $\sin ^{2}(2 \bar{\theta}) N^{3} \gg 2 \sin ^{4}(\bar{\theta}) N^{2}$. Thus, $I_{\pi}(2 n T)$ scales with $n^{2} N^{3}$ in the large- $N$ limit, as shown in Figs. 3(g) and 3(h). Correspondingly, $\Delta_{J T} \geqslant 1 / \sqrt{I_{\pi}(2 n T)} \sim n^{-1} N^{-3 / 2}$. This is precisely what we have obtained in Figs. 3(c) and 3(e).

The DTCs discussed in the literature are stable within a finite range of both the interaction strength and a uniform deviation of $\theta_{i}$ from $\pi$. In contrast, our DTC is stable against any spatial fluctuations in $\theta_{i}$ and while being supersensitive to $J T$. In practice, it is easier to control $J$ and $T$ than the $N$ local parameters $\theta_{i}$ in a noisy environment, where $\theta_{i}$ may not have any correlations at different locations. Moreover, our DTC could measure $J T$ with high precision beyond the Heisenberg limit. It mimics a supersensitive clock. If the frequency of the external field $\omega_{\mathrm{d}}=2 \pi / T$ is fixed, $J$ corresponds to some internal parameter of a clock (for instance, the length of a pendulum clock) and needs to be tuned with a precision of $1 / N^{3 / 2}$ to deliver rigid ticks at $t=2 n T$. Otherwise, as shown in Figs. 3(c) and 3(e), once $J T$ is beyond the length $1 / N^{3 / 2}$ centered at $J T=\pi$, both $P(2 T)$ and $\tilde{P}(f)$ quickly decrease and the DTC stalls to avoid errors in the timekeeping. From $J T=\pi$, the precision of $J$ can be estimated as $\Delta_{J} / J \approx$ $\Delta_{\mathrm{d}} / \omega_{\mathrm{d}}+N^{-3 / 2}$, where $\Delta_{\mathrm{d}} / \omega_{\mathrm{d}}$ characterizes the precision of the driving frequency. When $N^{-3 / 2} \gg \Delta_{\mathrm{d}} / \omega_{\mathrm{d}}, \Delta_{J} / J$ scales with $N^{-3 / 2}$. When $N \rightarrow\left(\Delta_{\mathrm{d}} / \omega_{\mathrm{d}}\right)^{-2 / 3}$, the uncertainty of $J$ approaches the precision limit of $\Delta_{\mathrm{d}} / \omega_{\mathrm{d}}$. Whereas the precision of $\Delta_{\mathrm{d}} / \omega_{\mathrm{d}}$ is up to $10^{-19}$ in the terahertz regime [35], typical experiments on ultracold atoms, ion traps, and nitrogenvacancy centers have interaction strengths $\sim 10^{2}-10^{5} \mathrm{~Hz}$. In such a regime, the precision of $\Delta_{\mathrm{d}} / \omega_{\mathrm{d}}$ could be $10^{-6}$ and higher. Our results thus provide an application of precision timekeeping in many-body physics.

Fixing $J$, our DTC could also gauge the frequency. Only when $T$ deviates from $\pi / J$ within $1 / N^{3 / 2}$ could a driving field induce a long-lasting dynamics. Unlike atomic clocks using a narrow linewidth transition, here many-body effects determine the driving frequency. The rotated angle $\bar{\theta}$ is arbitrary such that this DTC functions in a nonideal environment, unlike previous works requiring precise control of pulses in nonlinear metrology without periodic driving $[29,30,36]$.

We have also found that $M_{z}\left(2 T^{-}\right)$and $\tilde{M}\left(\frac{1}{2 T}\right)$ scale with $1 / N^{1 / 2}$ and $1 / N$, as shown in Figs. 3(b), 3(d), and 3(f). Similar scalings are obtained for other uniform rotations. For instance, when $\theta_{i}=\pi / 2, \Delta_{J T}$ of either $\tilde{P}\left(\frac{1}{2 T}\right)$ or $\tilde{M}_{z}\left(\frac{1}{2 T}\right)$ scales with $1 / N$ (Appendix C).

Previous conclusions apply to a generic long-range interaction if its range is much larger than the size of the system. For instance, with decreasing $\alpha$, the range of a power-law potential in Eq. (6) increases. When $\alpha=0$, it is equivalent to the all-to-all interaction. Figure 4 shows results for $N=14$. With decreasing $\alpha$ down to zero, $P(2 n T)$ and $M_{z}(2 n T)$ increase and approach the result of the all-to-all interaction. A small $\alpha=0.04$ readily provides us with a good approximation of the all-to-all interaction in such a finite system.

Both interactions and external drivings are crucial for DTCs. We hope that our work will stimulate more studies 


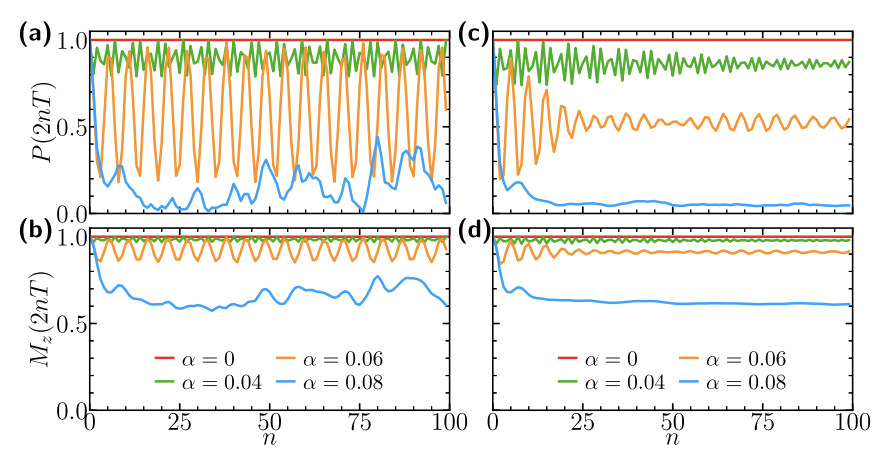

FIG. 4. Power-law potentials for $N=14$. (a) and (b) Uniform rotations with $\bar{\theta}=0.95 \pi$. Here $\alpha=0$ corresponds to the all-to-all interaction. With decreasing $\alpha$, the results of the power-law potentials approach those of the all-to-all interaction. Other parameters are the same in (a) and (b). (c) and (d) Inhomogeneous rotations with $\bar{\theta}=\pi$ and $w_{s}=0.1 \pi$.

of their interplay to access different nonequilibrium quantum states with long coherent times.

\section{ACKNOWLEDGMENTS}

We acknowledge C.-L. Hung for helpful discussions on the precision of measuring frequencies. This work was supported by DOE Grant No. DE-SC0019202, W. M. Keck Foundation, and the Center for Science of Information, a NSF Science and Technology Center, under Grant Agreement No. CCF0939370. C.Lv acknowledges support from Purdue Research Foundation.

\section{APPENDIX A: ON-SITE DISORDER}

The on-site disorder is often considered in a DTC to introduce many-body localization. Since the coupling between $l$-bits decays exponentially with increasing their distance, this could slow down the thermalization, provided $\theta_{i}$ is spatially uniform. However, this mechanism of suppressing the thermalization automatically weakens the synchronization between different spatial parts of the system. Thus, when $\theta_{i}$ has strong spatial inhomogeneities, the on-site disorder cannot stabilize the DTC. Consider the Hamiltonian

$$
\begin{aligned}
H= & 2 J \sum_{i<j} \frac{S_{i}^{z} S_{j}^{z}}{|i-j|^{\alpha}}+\sum_{n} \delta(t-n T) \sum_{i=1}^{N} \theta_{i} S_{i}^{y} \\
& +2 \sum_{i=1}^{N} \Delta_{i} S_{i}^{z},
\end{aligned}
$$
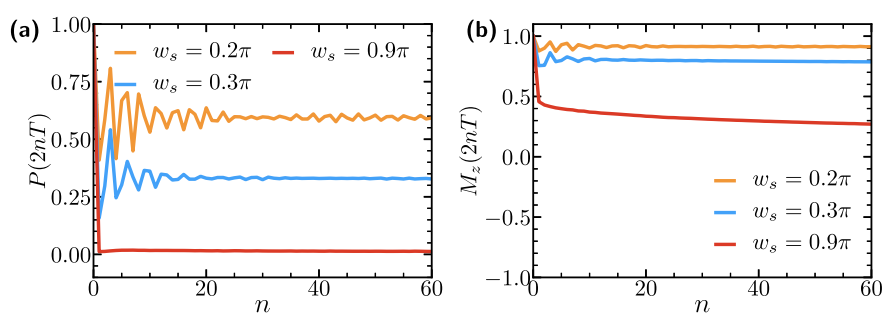

where $\theta_{i}$ has a uniform distribution in $\left[\bar{\theta}-w_{s}, \bar{\theta}+w_{s}\right]$, similar to the main text. The on-site disorder $\Delta_{i}$ has a uniform distribution in $[0, W]$. As shown in Fig. 5, for a given $W$, with increasing $w_{s}, P(2 n T)$ and $L_{z}(2 n T)$ are suppressed down to zero. Meanwhile, the entropies $S(2 n T)$ and $Q(2 n T)$ grow faster, signifying the thermalization of the DTC.

\section{APPENDIX B: INHOMOGENEOUS PULSES WITH $\bar{\theta} \neq \pi$}

In Fig. 2 we have shown the results of inhomogeneous pulses with $\bar{\theta}=\pi$. Here we present the results when $\bar{\theta} \neq$ $\pi$. As shown in Figs. 6(a)-6(d), when $\bar{\theta}=0.9 \pi, P$ and $M_{z}$ are suppressed more quickly and $S$ and $Q$ grow faster than the results for $\bar{\theta}=\pi$. If $\bar{\theta}$ decreases further down to $0.8 \pi$, this tendency continues, showing that the power interaction cannot synchronize spins when pulses are not uniform. In contrast, the results of the all-to-all interaction remain unchanged.

\section{APPENDIX C: SCALINGS WITH PARTICLE NUMBERS}

\section{Scalings at $t=2 T$}

We have analytically obtained how the dependence of $P(2 T)\left[M_{z}(2 T)\right]$ on $J T$ scales with the particle number $N$,

$$
\begin{aligned}
P^{\prime}(\delta) \equiv & P(2 T ; J T=\pi+\delta) \\
= & \left(1+\frac{N^{2} \delta^{2} \sin ^{4} \theta}{4}\right)^{-1 / 2} \\
& \times \exp \left(-\frac{1}{4 N^{2} \delta^{2} \sin ^{4} \theta+16} \sin ^{2}(2 \theta) \delta^{2} N^{3}\right), \\
M_{z}^{\prime}(\delta) \equiv & M_{z}(2 T ; J T=\pi+\delta) \\
= & \cos ^{2} \theta+\sin ^{2} \theta \cos (\cos \theta N \delta) e^{-(N / 2) \delta^{2} \sin ^{2} \theta} .
\end{aligned}
$$

When $\theta=\pi / 2, \sin (2 \theta)=0$. The exponential function in Eq. $(\mathrm{C} 1)$ becomes an identity and $P^{\prime}(\delta)=\left(1+\frac{N^{2} \delta^{2} \sin ^{4} \theta}{4}\right)^{-1 / 2}$. Thus, the peak width shown in Fig. 7 scales with $1 / N$. The same scaling applies to $\theta$ near 0 and $\pi$. In contrast, when $\theta$ is away from $0, \pi / 2$, and $\pi$, the exponential function becomes dominant and $P(2 T)$ decays faster, as shown in Fig. 3(c). In particular, the peak width of $P(2 T)$ in Fig. 3(e) scales with $1 / N^{3 / 2}$. In Eq. (C2) the $N \delta$ term in the cosine function leads to a fast oscillation and the $N \delta^{2}$ term in the exponential function leads to the $1 / N^{1 / 2}$ scaling of the profile of $M_{z}(2 T)$, regardless of $\theta$, as shown in the insets of Figs. 7(d) and 3(d).

To derive Eqs. (C1) and (C2), we consider an initial state $\left|\Psi\left(0^{-}\right)\right\rangle=\prod_{i}|\uparrow\rangle_{i}=\left|\frac{N}{2}, \frac{N}{2}\right\rangle$, where $L_{z}\left|\frac{N}{2}, l\right\rangle=l\left|\frac{N}{2}, l\right\rangle$
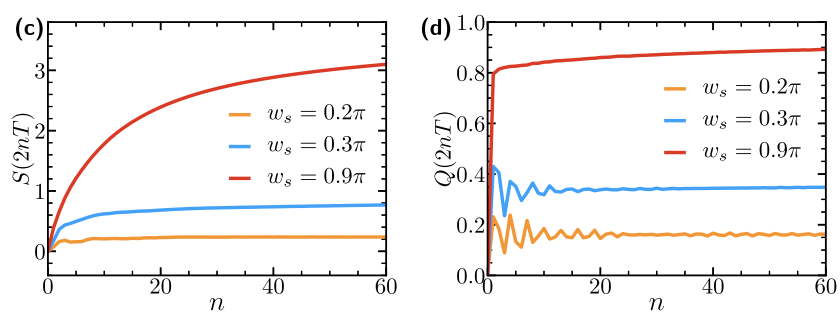

FIG. 5. Effects of inhomogeneous $\theta_{i}$ on MBL. Here $\theta_{i}$ is chosen from a uniform distribution $\left[-w_{s}+\bar{\theta}, \bar{\theta}+w_{s}\right]$. The on-site disorder is chosen from $[0, W]$, where $W=40 J, \alpha=3, \bar{\theta}=\pi$, and $N=14$. 

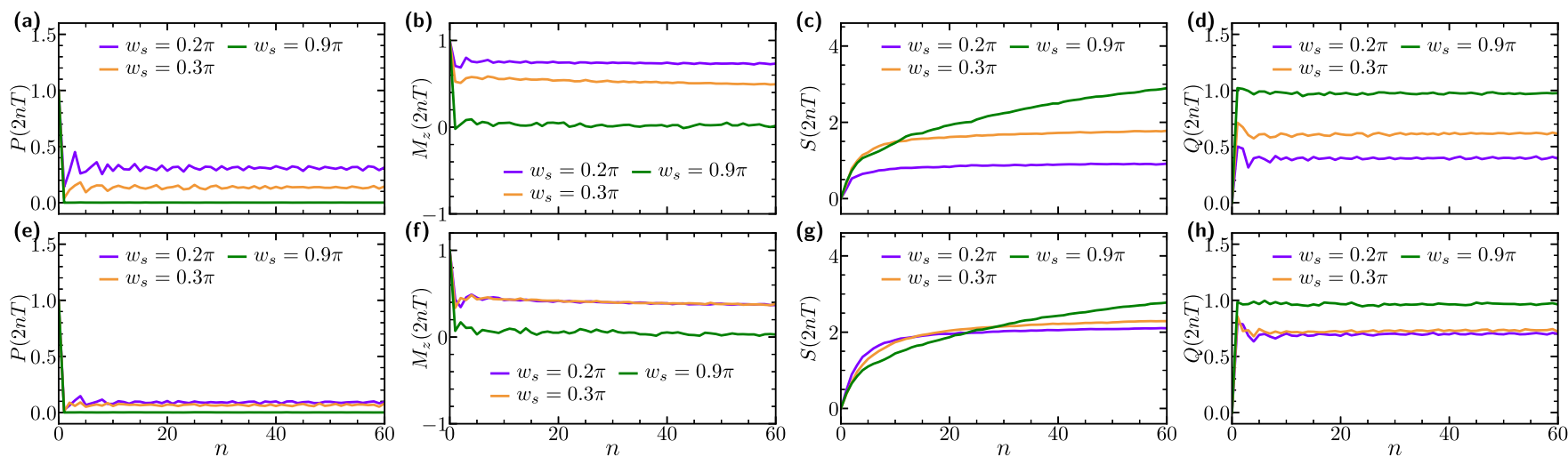

FIG. 6. Results of inhomogeneous pulses for a power-law potential with $\alpha=3$. The parameters are the same as in Figs. 2(e)-2(h) except here in (a) and (b) $\bar{\theta}=0.9 \pi$ and in (e)-(h) $\bar{\theta}=0.8 \pi$.

and $\frac{N}{2}=L$ is the total angular momentum

$$
\begin{aligned}
P^{\prime}(\delta)= & \mid\left\langle\Psi\left(0^{-}\right)\right| e^{-i(\pi+\delta) L_{z}^{2}} \\
& \times\left. e^{-i \theta L_{y}} e^{-i(\pi+\delta) L_{z}^{2}} e^{-i \theta L_{y}}\left|\Psi\left(0^{-}\right)\right\rangle\right|^{2} \\
= & \mid e^{-i(\pi+\delta)(N / 2)^{2}}\left\langle\Psi\left(0^{-}\right)\right| e^{-i \theta L_{y}} \\
& \times\left. e^{-i(\pi+\delta) L_{z}^{2}} e^{-i \theta L_{y}}\left|\Psi\left(0^{-}\right)\right\rangle\right|^{2} .
\end{aligned}
$$

As discussed in the main text, $e^{-i \pi L_{z}^{2}}=e^{-i \pi L_{z}}$ is satisfied for any even particle number $N$. When $\delta$ is small, $e^{-i \delta L_{z}^{2}}$ can be written as

$$
e^{-i \delta L_{z}^{2}} \approx \int_{-N \delta}^{N \delta} d k e^{-i k^{2} / 4 \delta} \frac{1}{2 \pi} \frac{\sqrt{\pi}}{\sqrt{-i \delta}} e^{-i k L_{z}} .
$$

We thus obtain

$$
P^{\prime}(\delta)=\left|e^{-i(\pi+\delta)(N / 2)^{2}} \int_{-N \delta}^{N \delta} d k e^{-i k^{2} / 4 \delta} \frac{1}{2 \pi} \frac{\sqrt{\pi}}{\sqrt{-i \delta}} I(\theta, k)\right|^{2},
$$

$$
I(\theta, k)=\left\langle\Psi\left(0^{-}\right)\left|e^{-i \theta L_{y}} e^{-i \pi L_{z}} e^{-i k L_{z}} e^{-i \theta L_{y}}\right| \Psi\left(0^{-}\right)\right\rangle .
$$
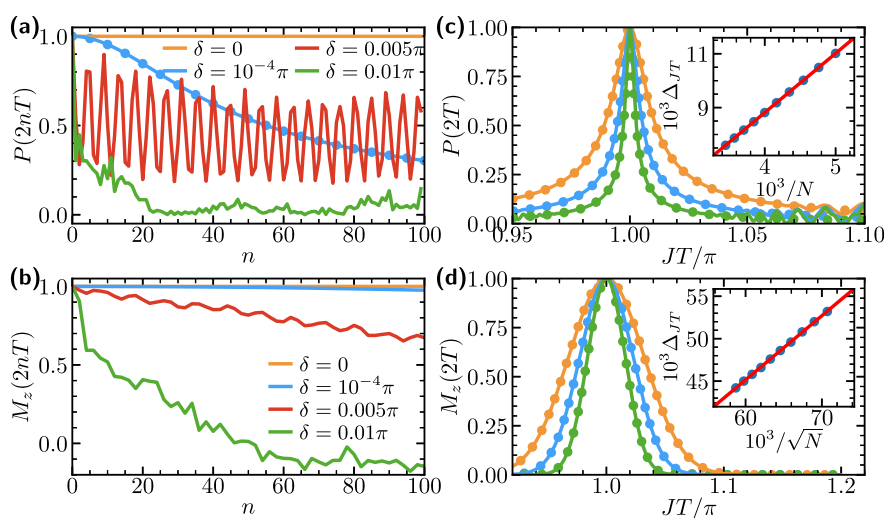

Noting that

$$
\begin{aligned}
e^{-i \theta L_{y}}\left|\Psi\left(0^{-}\right)\right\rangle & =|\theta, 0\rangle_{c}, \\
e^{i \pi L_{z}} e^{i \theta L_{y}}\left|\Psi\left(0^{-}\right)\right\rangle & =(-1)^{N / 2}|\theta, 0\rangle_{c},
\end{aligned}
$$

we obtain

$$
\begin{aligned}
I(\theta, k) & =(-1)^{N / 2}\left\langle\theta,\left.0\right|_{c} e^{-i k L_{z}} \mid \theta, 0\right\rangle_{c} \\
& =(-1)^{N / 2} e^{i k(N / 2)}\left(\frac{1}{1+\alpha}\right)^{N}\left(e^{-i k} \alpha+1\right)^{N},
\end{aligned}
$$

where $\alpha \equiv \tan ^{2} \frac{\theta}{2}$ and

$$
\begin{aligned}
|\theta, \phi\rangle_{c}= & \sum_{l=-N / 2}^{N / 2} \sqrt{\frac{N !}{\left(\frac{N}{2}+l\right) !\left(\frac{N}{2}-l\right) !}} \\
& \times(\cos \theta)^{N / 2+l}(\sin \theta)^{N / 2-l} e^{i \phi(N / 2-l)}|l\rangle
\end{aligned}
$$
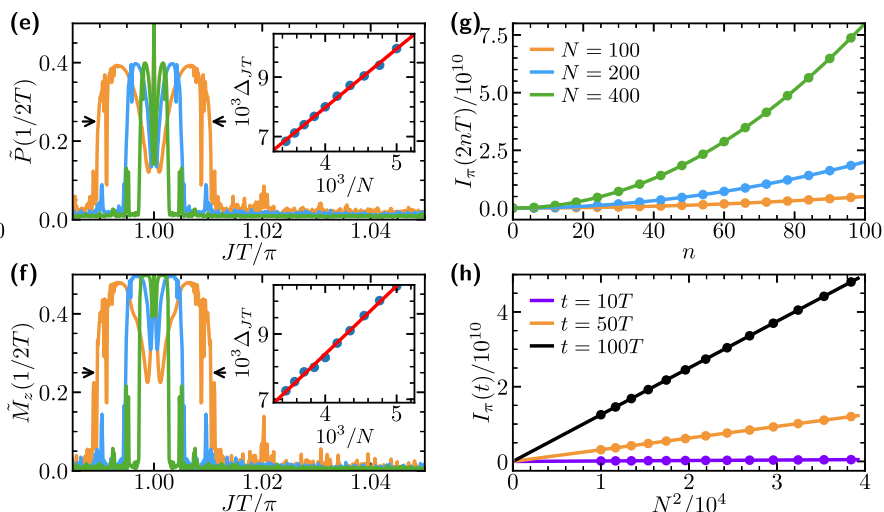

FIG. 7. Sensitivity of the DTC to $J T$ when $\theta=\pi / 2$. Dots are analytical results and curves are the numerical results. Orange, blue, and green colors in (c)-(f) represent $N=100,200$, and 400, respectively. (a) $P(2 n T)$ and (b) $M_{z}(2 n T)$ as functions of $n$ for various $J T$. When $|J T-\pi| \gg \pi / N$ (here $N=200$ ), both quantities quickly decrease down to zero. (c) $P(2 T)$ and (d) $M_{z}(2 T)$ at a fixed time $t=2 T$ as functions of $J T$. For a fixed $N$, both quantities are featured with narrow peaks centered at $J T=\pi$. Insets show the scalings of the widths $\Delta$ (full width at half maximum) of the peaks with $N$. Power spectra (e) $\tilde{P}(1 / 2 T)$ and (f) $\tilde{M}_{z}(1 / 2 T)$ are also featured with narrow peaks around $J T=\pi$. Whereas they exhibit nonmonotonic behaviors near $J T=\pi$, both quantities vanish when $|J T-\pi| \gg \pi / N$. Insets show the scalings of the widths of the peaks with $N$. (g) Quantum Fisher information $I_{\pi}(2 n T)$ as a function of $n$. (h) $I_{\pi}(2 n T)$ is proportional to $N^{2}$. The value $\theta_{i}=\pi / 2$ is used in all panels. 


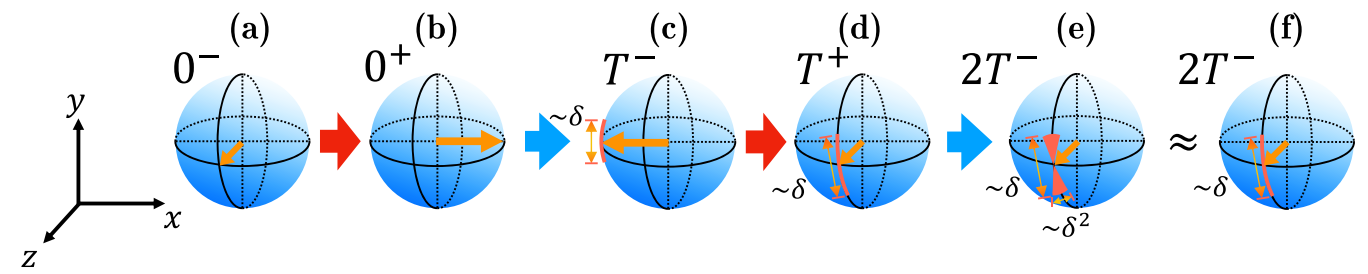

FIG. 8. Approximation used to derive $P(2 n T)$. (a) and (b) The states before and after the first pulse. (c) When $t=T^{-}$, the nonlinear operator $e^{-i J T L_{z}^{2}}$ creates a superposition of coherent states, which spans a length scale $\gamma$ in the latitude direction. (d) This length scale is transferred to the longitude direction around the north pole by the pulse at $t=T^{+}$. Then the nonlinear operator creates a superposition of coherent states in the region highlighted by the red color at $t=2 T^{-}$. (e) The length scales of this region in the longitude and latitude directions are $\delta$ and $\delta^{2}$, respectively. (f) Replacing the second nonlinear operator $e^{-i J T L_{z}^{2}}$ by $e^{-i \pi L_{z}^{2}}$, we have ignored the expansion of the wave function in the latitude direction that gives rise to a high-order correction to $P(2 n T)$ at small times.

is a coherent state pointing along $\theta$ and $\phi$. In the large- $N$ limit,

$$
\begin{aligned}
I(\theta, k) \approx & (-1)^{N / 2} \exp \left(-i k \frac{N}{2}+i \frac{N}{1+\alpha} k\right) \\
& \times \exp \left(-\frac{N}{2} \frac{\alpha}{(1+\alpha)^{2}} k^{2}\right),
\end{aligned}
$$

which represents a narrow Gaussian centered at $k=0$. Substituting $I(\theta, k)$ in Eq. (C5) using Eq. (C11), we obtain Eq. (C1).

As for $M_{z}^{\prime}(\delta)$, using the time evolution operator $U(T)=$ $e^{-i L_{z}^{2} J T} e^{-i L_{y} \theta}$, we obtain the Heisenberg equations, which provide us with the nonlinear recursion relations as shown in [27],

$$
\begin{aligned}
L_{x}^{\prime}= & U^{-1}(T) L_{x} U(T)=\frac{1}{2}\left(L_{x} \cos \theta+L_{z} \sin \theta+i L_{y}\right) \\
& \times e^{i 2 J T\left(L_{z} \cos \theta-L_{x} \sin \theta+1 / 2\right)+\text { H.c. },} \\
L_{y}^{\prime}= & U^{-1}(T) L_{y} U(T)=\frac{1}{2 i}\left(L_{x} \cos \theta+L_{z} \sin \theta+i L_{y}\right) \\
& \times e^{i 2 J T\left(L_{z} \cos \theta-L_{x} \sin \theta+1 / 2\right)}+\text { H.c. }, \\
L_{z}^{\prime}= & U^{-1}(T) L_{z} U(T)=L_{z} \cos \theta-L_{x} \sin \theta .
\end{aligned}
$$

Since

$$
\begin{aligned}
M_{z}(2 T)= & \frac{2}{N}\left\langle\Psi\left(0^{-}\right)\right| U^{-1}(T) \\
& \times U^{-1}(T) L_{z} U(T) U(T)\left|\Psi\left(0^{-}\right)\right\rangle,
\end{aligned}
$$

we obtain

$$
\begin{aligned}
M_{z}^{\prime}(\delta) & =\frac{2}{N}\left\langle\Psi\left(0^{-}\right)\left|\left(L_{z}^{\prime} \cos \theta-L_{x}^{\prime} \sin \theta\right)\right| \Psi\left(0^{-}\right)\right\rangle \\
& =\cos ^{2} \theta-(-1)^{N / 2} \sin \theta\left(\sin \theta \cos ^{N} \frac{\Theta}{2} \cos (J T-N \Phi)+(\cos \theta+1) \cos ^{N} \frac{\Theta}{2} \tan \frac{\Theta}{2} \cos (\Phi+J T-N \Phi)\right) \\
& =\cos ^{2} \theta+(-1)^{N / 2} \sin \theta \cos ^{N} \frac{\Theta}{2}\left(\sin \theta \cos (\delta-N \Phi)+(\cos \theta+1) \tan \frac{\Theta}{2} \cos (\Phi+\delta-N \Phi)\right) \\
& \approx \cos ^{2} \theta+\sin ^{2} \theta \cos (\cos \theta N \delta) e^{-(N / 2) \delta^{2} \sin ^{2} \theta}
\end{aligned}
$$

where

$$
\begin{aligned}
& \Theta=\arccos \left(\cos ^{2} \theta+\cos 2 \delta \sin ^{2} \theta\right), \\
& \Phi=\arctan \left(\frac{-\sin \theta \sin (2 \delta)}{\cos \theta \sin \theta(-1+\cos 2 \delta)}\right) .
\end{aligned}
$$

The expression which contains $\Theta$ and $\Phi$ is exact for any $\theta$ and $\delta$. The final approximation comes from $\Theta^{2}=4 \sin ^{2} \theta \delta^{2}+O\left(\delta^{4}\right), \quad \Phi=\pi / 2-\cos \theta \delta+O\left(\delta^{3}\right)$, and $\cos ^{N} \frac{\Theta}{2} \approx e^{-N \delta^{2} \sin ^{2} \theta / 2}$ when $\delta$ is small and $N$ is large. The overall profile as shown in Fig. 3(d) is thus given by $e^{-(N / 2) \sin ^{2} \theta \delta^{2}} \sin ^{2} \theta+\cos ^{2} \theta$.

\section{Scalings of $\tilde{P}\left(\frac{1}{2 T}\right)$}

We have also obtained an analytical form for $\tilde{P}(f)$, the Fourier transform of $P(2 n T)$. As shown in Fig. 8, starting from an initial state at the north pole, the state at $t=2 T^{-}$ covers a finite small region near the north pole if $\delta=J T-\pi$ is small. The length scales of the longitude and latitude directions are proportional to $\delta$ and $\delta^{2}$, the latter of which can be ignored in the small- $\delta$ limit. Thus, we make use of the following approximation to capture the dynamics in the small- $\delta$ limit:

$$
\begin{aligned}
U(2 T) & =e^{-i J T L_{z}^{2}} e^{-i \theta L_{y}} e^{-i J T L_{z}^{2}} e^{-i \theta L_{y}} \\
& \approx e^{-i \pi L_{z}^{2}} e^{-i \theta L_{y}} e^{-i J T L_{z}^{2}} e^{-i \theta L_{y}} .
\end{aligned}
$$


Here $P(2 n T)$ is written as

$$
\begin{aligned}
P(2 n T)= & \mid\left\langle\Psi\left(0^{-}\right)\right|\left(e^{-i \pi L_{z}^{2}}\right. \\
& \left.\times e^{-i \theta L_{y}} e^{-i(\pi+\delta) L_{z}^{2}} e^{-i \theta L_{y}}\right)\left.^{n}\left|\Psi\left(0^{-}\right)\right\rangle\right|^{2} .
\end{aligned}
$$

Using the identities $e^{-i \pi L_{z}} e^{-i \theta L_{y}} e^{-i \pi L_{z}}=e^{i \theta L_{y}}$ and $e^{-i \pi L_{z}^{2}}=$ $e^{-i \pi L_{z}}$, Eq. (C17) can be written as

$$
\begin{aligned}
P(2 n T) & =\left|\left\langle\Psi\left(0^{-}\right)\left|\left(e^{i \theta L_{y}} e^{-i \delta L_{z}^{2}} e^{-i \theta L_{y}}\right)^{n}\right| \Psi\left(0^{-}\right)\right\rangle\right|^{2} \\
& =\left|\left\langle\Psi\left(0^{-}\right)\left|e^{i \theta L_{y}} e^{-i n \delta L_{z}^{2}} e^{-i \theta L_{y}}\right| \Psi\left(0^{-}\right)\right\rangle\right|^{2} .
\end{aligned}
$$

Applying Eq. (C1), we obtain

$$
\begin{aligned}
P(2 n T)= & \exp \left(-\frac{n^{2} \sin ^{2}(2 \theta) \delta^{2} N^{3}}{4 N^{2} n^{2} \delta^{2} \sin ^{4} \theta+16}\right) \\
& \times\left(1+\frac{n^{2} N^{2} \delta^{2} \sin ^{4} \theta}{4}\right)^{-1 / 2} .
\end{aligned}
$$

Equation (C19) recovers Eq. (C1) when $n=1$. As shown in Fig. 7(a), this expression captures well the initial decay of $P(2 n T)$. However, it cannot describe the revival of $P(2 n T)$ at later times for certain $J T$ due to the approximation made in Eq. (C16).

The power spectrum is therefore written as

$$
\begin{aligned}
\tilde{P}(1 / 2 T) & =\frac{1}{M} \sum_{n=0}^{M-1} P(n T) e^{i(2 \pi / 2 T) n T} \\
& =\frac{1}{M} \sum_{n=0}^{M-1} P(n T)(-1)^{n} \\
& \approx \frac{1}{M} \sum_{n=0}^{M / 2-1} P(2 n T)
\end{aligned}
$$

where $M$ is the cutoff required in numerics. In the last step, we have used the fact that, for small $n,|\psi(2 n T+T)\rangle$ is located at a place on the Bloch sphere away from the north pole, provided $\theta$ is not small, and thus $P(2 n T+T)=$ $\left|\left\langle\Psi\left(0^{-}\right)|| \psi(2 n T+T)\right\rangle\right|^{2} \approx 0$.

When $n N \delta \ll 1$ and $\theta \neq 0, \pi / 2, \pi$, Eq. (C19) becomes $P(2 n T)=e^{-n^{2} \sin ^{2}(2 \theta) \delta^{2} N^{3} / 16}$ and Eq. (C20) is rewritten as

$$
\tilde{P}(1 / 2 T) \approx \frac{2 \sqrt{\pi} \operatorname{erf}\left(\frac{1}{8} \delta M N^{3 / 2} \sin 2 \theta\right)}{\sin (2 \theta) \delta M N^{3 / 2}} .
$$

In the limit $M \rightarrow \infty, \tilde{P}(1 / 2 T) \rightarrow \frac{1}{2 \sqrt{\pi}} e^{-\delta^{2} N^{3} M^{2} / 64}$. To compare with numerical result, we choose $\theta=\pi / 4$ and $M=200$. Equation (C21) becomes

$$
\tilde{P}(1 / 2 T) \approx \frac{2 \sqrt{\pi} \operatorname{erf}\left(25 \delta N^{3 / 2}\right)}{200 \delta N^{3 / 2}}
$$

which shows the $1 / N^{3 / 2}$ scaling. Here erf is the error function. The comparison between this analytical result and the numerical one is shown in Fig. 3(e).

When $\theta=\pi / 2$, the exponential term in Eq. (C19) becomes an identity. We obtain

$$
\tilde{P}(1 / 2 T) \approx \frac{\operatorname{arcsinh}(\delta M N / 4)}{\delta M N / 2} .
$$

As mentioned in Fig. 7, when $\theta=\pi / 2$, the dependence of $\tilde{P}(1 / 2 T)$ on $J T$ is not monotonic. With increasing $\delta, \tilde{P}(1 / 2 T)$ first quickly decreases and then increases before it eventually vanishes when $\delta>\pi / N$. Equation (C23) captures the narrow peak, whose width is much smaller than $\pi / N$, near $\delta=0$. The broader peak scales with $1 / N$ as shown in Fig. 7(f). When $\theta$ deviates from $\pi / 2$, the broader peak gets suppressed as shown in Fig. 9. When $\theta=\pi / 4$, only the central narrow peak is visible, whose width scales with $1 / N^{3 / 2}$, as discussed before.

Here $M_{z}(2 n T)$ and $\tilde{M}_{z}(1 / 2 T)$ do not have simple analytical forms. We have numerically evaluated them and the scaling of $\tilde{M}_{z}(1 / 2 T)$ with $N$ is shown in Figs. 7(b) and 7(e).
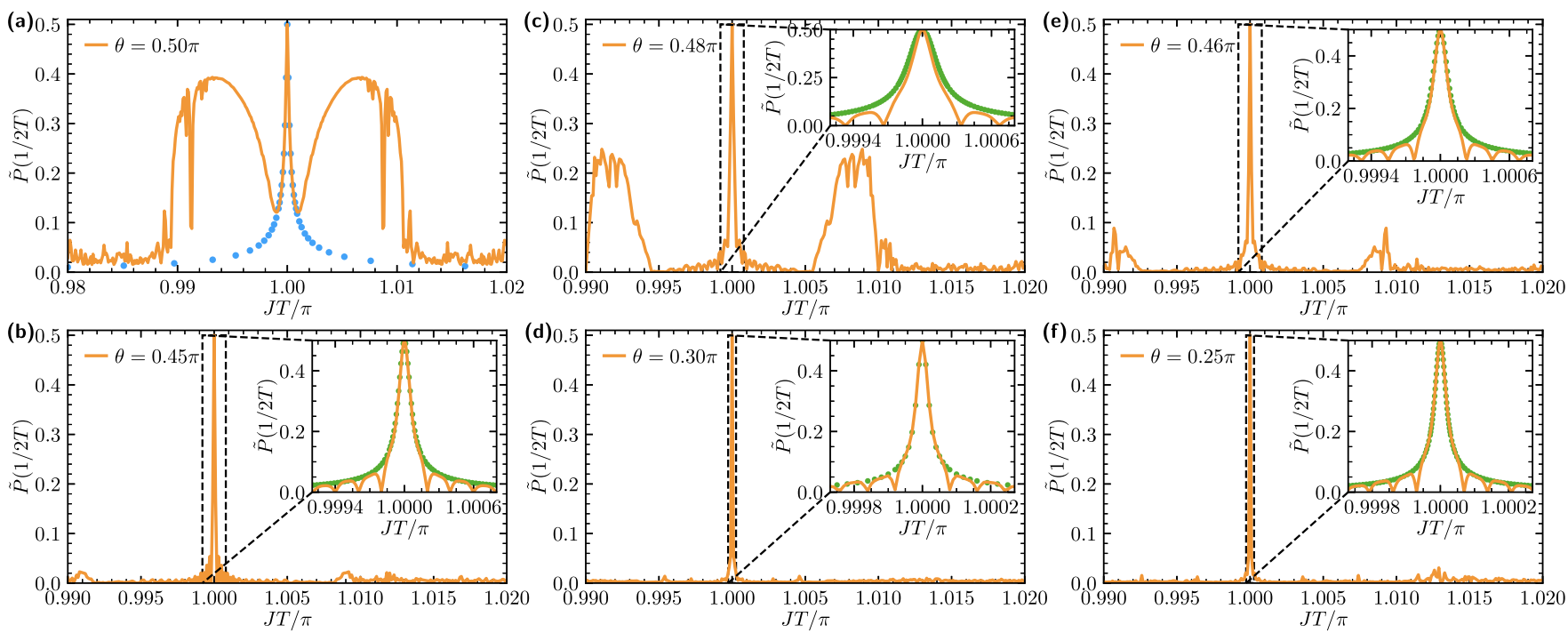

FIG. 9. Power spectra $\tilde{P}(1 / 2 T)$ for different pulses for $N=100$ and $M=200$. (a) Identical to the curve for $N=100$ in Fig. 7(e). The central sharp peak at $J T=\pi$ for the $\pi / 2$ pulse is well approximated by the analytical result (blue dots) shown in Eq. (C23). (b)-(f) When $\theta$ decreases, the two broader peaks gradually vanish. When the exponential term in Eq. (C19) dominates, the central peak is described by Eq. (C21) (green dots). Enlarging (f) around $J T=\pi$ gives rise to Fig. 3(e). 


\section{APPENDIX D: QUANTUM FISHER INFORMATION}

When $J T=\pi$, the quantum Fisher information is written as

$$
\begin{gathered}
I_{\pi}(2 n T)=\lim _{\epsilon \rightarrow 0} 4 \frac{1-F_{\epsilon}}{\epsilon^{2}}, \\
F_{\epsilon}=\left|\left\langle\Psi\left(0^{-}\right)\left|U_{\pi}(-2 n T) U_{\pi+\epsilon}(2 n T)\right| \Psi\left(0^{-}\right)\right\rangle\right|^{2} .
\end{gathered}
$$

As $\quad U_{\pi}(2 n T)\left|\Psi\left(0^{-}\right)\right\rangle=\left|\Psi\left(0^{-}\right)\right\rangle, \quad$ or equivalently $\left\langle\Psi\left(0^{-}\right)\right| U_{\pi}(-2 n T)=\left\langle\Psi\left(0^{-}\right)\right|$, the Loschmidt echo is identical to the quantum memory of the initial state $F_{\epsilon}=\left|\left\langle\Psi\left(0^{-}\right)\left|U_{\pi+\epsilon}(2 n T)\right| \Psi\left(0^{-}\right)\right\rangle\right|^{2}$. Using Eq. (C19) and replacing $\epsilon$ by $\delta$, we obtain

$$
\begin{aligned}
I_{\pi}(2 n T) & =\lim _{\delta \rightarrow 0} 4 \frac{1-P(2 n T)}{\delta^{2}} \\
& =\frac{n^{2} N^{3} \sin ^{2}(2 \theta)}{4}+\frac{n^{2} N^{2} \sin ^{4} \theta}{2} .
\end{aligned}
$$

\section{APPENDIX E: NUMERICAL SIMULATION METHODS}

In this Appendix we present some details of the numerical simulations used to produce Figs. 2, 3, and 7. We write the time-dependent many-body wave function $|\Psi(t)\rangle$ as a superposition of Fock states, which are eigenstates of $S_{z}$. In the presence of $H_{\text {int }}$, every Fock state acquires a dynamical phase factor. Then $H_{\text {pul }}$ is applied to flip the spins at $t=n T$ to obtain the evolution of the many-body wave function.

The entanglement entropy $S$ in Fig. 2 is calculated by first tracing out half of the system and obtaining a density matrix $\rho_{B}$ for the other half of the system. We then diagonalize $\rho_{B}$ and obtain all its eigenvalues $v_{i}$. Then $S$ is calculated by using $S=-\sum_{i} v_{i} \ln \left(v_{i}\right)$.

The power spectra shown in Figs. 3 and 7 are obtained as follows. First $P(t)$ and $M_{z}(t)$ are evaluated in the time interval between $t=0$ and $t=M T$, where $M$ represents the longest time we consider in the simulation. We then use $\tilde{P}(f)=$ $\frac{1}{M} \sum_{n=0}^{M-1} e^{i 2 \pi n T f} P(n T)$ to calculate the power spectra.

We use the full width at half maximum to characterize the width of power spectrum. Namely, when the value of $P$ or
$M_{z}$ is decreased to half of its maximum, we define twice the deviation of $J T$ from $\pi$ as the width.

\section{APPENDIX F: EXPERIMENTAL REALIZATION}

In this Appendix we discuss the experimental realization of our model. Whereas we used idealized $\delta$-like kicks in the discussions in the main text, our results can be straightforwardly generalized to pulses with finite widths. If we consider the Hamiltonian

$$
H= \begin{cases}2 J \sum_{i<j} S_{i}^{z} S_{j}^{z}, & n T<t<(n+1) T-\tau \\ \sum_{i}^{N} \frac{\theta_{i}}{\tau} S_{i}^{y}, & (n+1) T-\tau<t<(n+1) T,\end{cases}
$$

where $\tau$ represents the finite width, we only need to replace $J T=\pi$ by $J(T-\tau)=\pi$ and other results remain unchanged.

In a finite system, as shown in the main text, to qualitatively demonstrate our results for a system consisting of 14 spins, a power-law interaction width $\alpha=0.04$ readily provides us with a good approximation of the all-to-all interaction. In fact, the only requirement is that the range of interaction is much larger than the size of the system. Meanwhile, the all-to-all interaction can be accessed by using photons in cavities or waveguides to couple atoms at different locations.

We consider the Hamiltonian

$$
H=\hbar \omega b^{\dagger} b+\Omega\left(b^{\dagger}+b\right) \sum_{i}\left(S_{i}^{+}+S_{i}^{-}\right),
$$

where the first term represents a single-photon mode and the second term denotes the interaction between photons and atoms. We have assumed that the local Hamiltonian acting on each spin vanishes. In addition, $S_{i}^{ \pm}=S_{i}^{x} \pm S_{i}^{y}$. Without the second term, the eigenstates are $|n\rangle \prod_{i}\left|\eta_{i}\right\rangle$, representing $n$ photons and a Fock state of atoms, and $\eta=\uparrow, \downarrow$. Consider two specific atoms at sites $i \neq j$; their couplings induced by the photon can be derived using second-order perturbation. For instance, the off-diagonal term is written as

$$
\begin{aligned}
& \frac{\Omega^{2}\left\langle n\left|\left\langle\downarrow_{i} \uparrow_{j}\left|\prod_{k \neq i, j}\left\langle\eta_{k}\left|b S_{j}^{+}\right| n+1\right\rangle\right| \downarrow_{i} \downarrow_{j}\right\rangle \prod_{k \neq i, j}\right| \eta_{k}\right\rangle\left\langle n+1\left|\left\langle\downarrow_{i} \downarrow_{j}\left|\prod_{k \neq i, j}\left\langle\eta_{k}\left|b^{\dagger} S_{i}^{-}\right| n\right\rangle\right| \uparrow_{i} \downarrow_{j}\right\rangle \prod_{k \neq i, j}\right| \eta_{k}\right\rangle}{-\hbar \omega} \\
& \quad+\frac{\Omega^{2}\left\langle n\left|\left\langle\downarrow_{i} \uparrow_{j}\left|\prod_{k \neq i, j}\left\langle\eta_{k}\left|b^{\dagger} S_{j}^{+}\right| n-1\right\rangle\right| \downarrow_{i} \downarrow_{j}\right\rangle \prod_{k \neq i, j}\right| \eta_{k}\right\rangle\left\langle n-1\left|\left\langle\downarrow_{i} \downarrow_{j}\left|\prod_{k \neq i, j}\left\langle\eta_{k}\left|b S_{i}^{-}\right| n\right\rangle\right| \uparrow_{i} \downarrow_{j}\right\rangle \prod_{k \neq i, j}\right| \eta_{k}\right\rangle}{\hbar \omega}=-\frac{\Omega^{2}}{\hbar \omega} .
\end{aligned}
$$

This is equivalent to adding a term $-\frac{\Omega^{2}}{\hbar \omega} S_{j}^{+} S_{i}^{-}$to the unperturbed Hamiltonian. The diagonal couplings can be obtained by a similar means. The full effective Hamiltonian after eliminating the photon mode is written as

$$
H_{\mathrm{eff}}=-\frac{\Omega^{2}}{\hbar \omega} \sum_{i, j} S_{j}^{+} S_{i}^{-}+\text {H.c. }
$$

which can be further simplified as

$$
H_{\mathrm{eff}}=-\frac{2 \Omega^{2}}{\hbar \omega}\left(\sum_{i} \vec{S}_{i}\right)^{2}+\frac{2 \Omega^{2}}{\hbar \omega} \sum_{i}\left(S_{i}^{z}\right)^{2}+\frac{4 \Omega^{2}}{\hbar \omega} \sum_{i<j} S_{i}^{z} S_{j}^{z} .
$$

Since $\sum_{i} \vec{S}_{i}$ is conserved, the first two terms commute with the last term and the last term exactly matches an all-to-all 
interaction; we thus created an all-to-all interaction with an equivalent strength $J=\frac{2 \Omega^{2}}{\hbar \omega}$. Whereas the above discussion does not require the leakage of photons from the cavity, a "bad" cavity with leaking photons has the unique advantage of suppressing the heating that may be caused by the driving [37]. Thus, our results can be generalized to the full model including both the atoms and photons.

Whereas the above scheme is relevant to small systems, in which a fine-tuning of the local effective magnetic field is doable, our model can also be implemented by a two-mode bosonic system, in which the all-to-all interaction naturally exists. For instance, we consider a Bose-Einstein condensate in a double-well potential

$$
\begin{aligned}
H= & g_{1} \frac{n_{l}\left(n_{l}-1\right)+n_{r}\left(n_{r}-1\right)}{2} \\
& +g_{2} n_{l} n_{r}+\sum_{n}\left(\theta a_{l}^{\dagger} a_{r}+\text { H.c. }\right) \delta(t-n T),
\end{aligned}
$$

where $g_{1}$ and $g_{2}$ represent on-site and intersite interactions, respectively, and $\theta$ denotes the tunneling. If we map the left and the right site to the spin up and the spin down, respectively, $J=g_{1}+g_{2}$ directly corresponds to the all-to-all interaction, and the tunneling term is mapped to $H_{\text {pul }}$. Such a mapping allows us to implement all results in the spin model to a large bosonic system with $10^{4}$ particles and more.
[1] F. Wilczek, Quantum Time Crystals, Phys. Rev. Lett. 109, 160401 (2012).

[2] T. Li, Z.-X. Gong, Z.-Q. Yin, H. T. Quan, X. Yin, P. Zhang, L.-M. Duan, and X. Zhang, Space-time Crystals of Trapped Ions, Phys. Rev. Lett. 109, 163001 (2012).

[3] H. Watanabe and M. Oshikawa, Absence of Quantum Time Crystals, Phys. Rev. Lett. 114, 251603 (2015).

[4] K. Sacha, Modeling spontaneous breaking of time-translation symmetry, Phys. Rev. A 91, 033617 (2015).

[5] V. Khemani, A. Lazarides, R. Moessner, and S. L. Sondhi, Phase Structure of Driven Quantum Systems, Phys. Rev. Lett. 116, 250401 (2016).

[6] D. V. Else, B. Bauer, and C. Nayak, Floquet Time Crystals, Phys. Rev. Lett. 117, 090402 (2016).

[7] N. Y. Yao, A. C. Potter, I.-D. Potirniche, and A. Vishwanath, Discrete Time Crystals: Rigidity, Criticality, and Realizations, Phys. Rev. Lett. 118, 030401 (2017).

[8] S. Choi, J. Choi, R. Landig, G. Kucsko, H. Zhou, J. Isoya, F. Jelezko, S. Onoda, H. Sumiya, V. Khemani, C. von Keyserlingk, N. Y. Yao, E. Demler, and M. D. Lukin, Observation of discrete time-crystalline order in a disordered dipolar many-body system, Nature (London) 543, 221 (2017).

[9] J. Zhang, P. W. Hess, A. Kyprianidis, P. Becker, A. Lee, J. Smith, G. Pagano, I.-D. Potirniche, A. C. Potter, A. Vishwanath, N. Y. Yao, and C. Monroe, Observation of a discrete time crystal, Nature (London) 543, 217 (2017).

[10] D. Abanin, W. D. Roeck, W. W. Ho, and F. Huveneers, A rigorous theory of many-body prethermalization for periodically driven and closed quantum systems, Commun. Math. Phys. 354, 809 (2017).

[11] S. Pal, N. Nishad, T. S. Mahesh, and G. J. Sreejith, Temporal Order in Periodically Driven Spins in Star-Shaped Clusters, Phys. Rev. Lett. 120, 180602 (2018).

[12] J. Rovny, R. L. Blum, and S. E. Barrett, Observation of Discrete-Time-Crystal Signatures in an Ordered Dipolar ManyBody System, Phys. Rev. Lett. 120, 180603 (2018).

[13] B. Huang, Y.-H. Wu, and W. V. Liu, Clean Floquet Time Crystals: Models and Realizations in Cold Atoms, Phys. Rev. Lett. 120, 110603 (2018).

[14] D. V. Else, C. Monroe, C. Nayak, and N. Y. Yao, Discrete time crystals, Annual Review of Condensed Matter Physics 11, 467 (2020).

[15] K. Sacha and J. Zakrzewski, Time crystals: A review, Rep. Prog. Phys. 81, 016401 (2017).
[16] L. D'Alessio and M. Rigol, Long-Time Behavior of Isolated Periodically Driven Interacting Lattice Systems, Phys. Rev. X 4, 041048 (2014).

[17] P. Ponte, A. Chandran, Z. Papić, and D. A. Abanin, Periodically driven ergodic and many-body localized quantum systems, Ann. Phys. (NY) 353, 196 (2015).

[18] A. Lazarides, A. Das, and R. Moessner, Equilibrium states of generic quantum systems subject to periodic driving, Phys. Rev. E 90, 012110 (2014).

[19] A. Pal and D. A. Huse, Many-body localization phase transition, Phys. Rev. B 82, 174411 (2010).

[20] C. W. von Keyserlingk, V. Khemani, and S. L. Sondhi, Absolute stability and spatiotemporal long-range order in Floquet systems, Phys. Rev. B 94, 085112 (2016).

[21] H. Lipkin, N. Meshkov, and A. Glick, Validity of many-body approximation methods for a solvable model, Nucl. Phys. 62, 188 (1965).

[22] C.-L. Hung, A. González-Tudela, J. I. Cirac, and H. J. Kimble, Quantum spin dynamics with pairwise-tunable, longrange interactions, Proc. Natl. Acad. Sci. USA 113, E4946 (2016).

[23] H. Ritsch, P. Domokos, F. Brennecke, and T. Esslinger, Cold atoms in cavity-generated dynamical optical potentials, Rev. Mod. Phys. 85, 553 (2013).

[24] A. Russomanno, F. Iemini, M. Dalmonte, and R. Fazio, Floquet time crystal in the Lipkin-Meshkov-Glick model, Phys. Rev. B 95, 214307 (2017).

[25] B. Yan, S. A. Moses, B. Gadway, J. P. Covey, K. R. A. Hazzard, A. M. Rey, D. S. Jin, and J. Ye, Observation of dipolar spinexchange interactions with lattice-confined polar molecules, Nature (London) 501, 521 (2013).

[26] L. D'Alessio and A. Polkovnikov, Many-body energy localization transition in periodically driven systems, Ann. Phys. (NY) 333, 19 (2013).

[27] F. Haake, M. Kuś, and R. Scharf, Classical and quantum chaos for a kicked top, Z. Phys. B 65, 381 (1987).

[28] S. Boixo, S. T. Flammia, C. M. Caves, and JM Geremia, Generalized Limits for Single-Parameter Quantum Estimation, Phys. Rev. Lett. 98, 090401 (2007).

[29] S. Choi and B. Sundaram, Bose-Einstein condensate as a nonlinear Ramsey interferometer operating beyond the Heisenberg limit, Phys. Rev. A 77, 053613 (2008).

[30] M. Napolitano, M. Koschorreck, B. Dubost, N. Behbood, R. J. Sewell, and M. W. Mitchell, Interaction-based quantum 
metrology showing scaling beyond the Heisenberg limit, Nature (London) 471, 486 (2011).

[31] R. J. Sewell, M. Napolitano, N. Behbood, G. Colangelo, F. Martin Ciurana, and M. W. Mitchell, Ultrasensitive Atomic Spin Measurements with a Nonlinear Interferometer, Phys. Rev. X 4, 021045 (2014).

[32] D. Braun, G. Adesso, F. Benatti, R. Floreanini, U. Marzolino, M. W. Mitchell, and S. Pirandola, Quantum-enhanced measurements without entanglement, Rev. Mod. Phys. 90, 035006 (2018).

[33] L. J. Fiderer and D. Braun, Quantum metrology with quantumchaotic sensors, Nat. Commun. 9, 1351 (2018).
[34] C. W. Helstrom, Quantum detection and estimation theory, J. Stat. Phys. 1, 231 (1969).

[35] G. E. Marti, R. B. Hutson, A. Goban, S. L. Campbell, N. Poli, and J. Ye, Imaging Optical Frequencies with $100 \mu \mathrm{Hz}$ Precision and $1.1 \mu \mathrm{m}$ Resolution, Phys. Rev. Lett. 120, 103201 (2018).

[36] A. M. Rey, L. Jiang, and M. D. Lukin, Quantum-limited measurements of atomic scattering properties, Phys. Rev. A 76, 053617 (2007).

[37] Z. Gong, R. Hamazaki, and M. Ueda, Discrete Time-Crystalline Order in Cavity and Circuit QED Systems, Phys. Rev. Lett. 120, 040404 (2018). 